\title{
Coulisses
}

Revue de théâtre

7 | Printemps 1993

Varia

\section{Rencontres avec Jacques Fornier}

Propos recueillis par Philippe Baron

Philippe Baron

\section{(2) OpenEdition}

Journals

Édition électronique

URL : http://journals.openedition.org/coulisses/2197

DOI : $10.4000 /$ coulisses. 2197

ISSN : 2546-9460

Éditeur

Presses universitaires de Franche-Comté

\section{Édition imprimée}

Date de publication : 1 avril 1993

Pagination : 18-21

ISSN : 1150-594X

\section{Référence électronique}

Philippe Baron, « Rencontres avec Jacques Fornier », Coulisses [En ligne], 7 | Printemps 1993, mis en ligne le 15 mars 2019, consulté le 24 octobre 2019. URL : http://journals.openedition.org/coulisses/ 2197 ; DOI : 10.4000/coulisses.2197

Ce document a été généré automatiquement le 24 octobre 2019.

Coulisses 


\title{
Rencontres avec Jacques Fornier
}

\author{
Propos recueillis par Philippe Baron
}

\author{
Philippe Baron
}

Le Centre de Rencontres de Besançon a la chance d'avoir comme directeur artistique Jacques FORNIER, ancien directeur du Théâtre de Bourgogne et du Théâtre National de Strasbourg. Jacques FORNIER coordonne tout l'enseignement théâtral qui est dispensé par ce centre et se charge lui-même de divers ateliers. Coulisses est allé l'interviewer.

Coulisses: Jacques fORNIER, pouvez-vous me dire d'où vous vient votre passion pour le théâtre?

Jacques FORNIER : Je suis issu d'une famille qui se partageait entre des activités très différentes. Un de mes oncles était général ; l'une de mes tantes, Rose RAY, qui avait été actrice à Paris entre les deux guerres mondiales m'a souvent entretenu de théâtre. J'ai eu divers emplois dans l'armée et dans le monde des finances. J'ai pensé que le théâtre réunissait tout et que, grâce au jeu théâtral, je pouvais être tout ce que dans la vie courante, il est difficile d'être à la fois.

- Je voudrais savoir quelle a été votre formation théâtrale.

- J'ai été élève à Paris d'un cours privé dirigé par un ancien acteur, Alick Roussel. J'y ai rencontré Roland BERTIN, actuellement sociétaire de la Comédie-Française.

- Je suppose que la formation était assez classique.

- Oui ; il nous faisait travailler la voix et le jeu en général.

- Quels rôles jouiez-vous le plus souvent?

- Les jeunes premiers, et surtout les valets, comme Figaro.

- Et lorsque vous avez quitté le cours?

- J'ai tout de suite voulu monter une compagnie avec Roland BERTIN et d'autres amis, Juliette BRAC, PAGES et François CHAUDAT.

- Vous vous êtes fixé...?

- En Bourgogne et nous avons pris le nom de Théâtre de Bourgogne. Nous étions très influencés par Jean VILAR ${ }^{1}$, Charles DULLIN et Jacques COPEAU.

Comme les Copiaux ${ }^{2}$ nous voulions révéler le théâtre à ceux qui le connaissaient peu. 
Nous pensions, comme VILAR, que lorsque ce public aurait conquis le théatre, des poètes naîtraient. C'était une petite erreur de VILAR.

- Vous logiez à...?

- A Pernand-Vergelesses où Marie-Hélène DASTE, la fille de Jacques COPEAU, nous a hébergés un an. Ensuite nous nous sommes installés à Beaune où nous partagions la même maison, comme les Copiaux à Morteuil.

- Vos ressources ne devaient pas être très grandes?

- Nous vivions uniquement des recettes que nous procuraient nos représentations. A cette époque, il n'existait pas d'Assedic pour les comédiens ${ }^{3}$. Nous avons joué cinquante fois la première année, et deux-cent-cinquante fois la seconde année.

- Quel était votre répertoire?

- Nous jouions beaucoup de Molière, notamment les farces. Nous jouions aussi du Musset.

- En somme le grand répertoire, à l'exemple de VILAR, pour qui le peuple est aristocrate et a droit au meilleur.

- Oui.

Jacques Fornier dans L'Avare

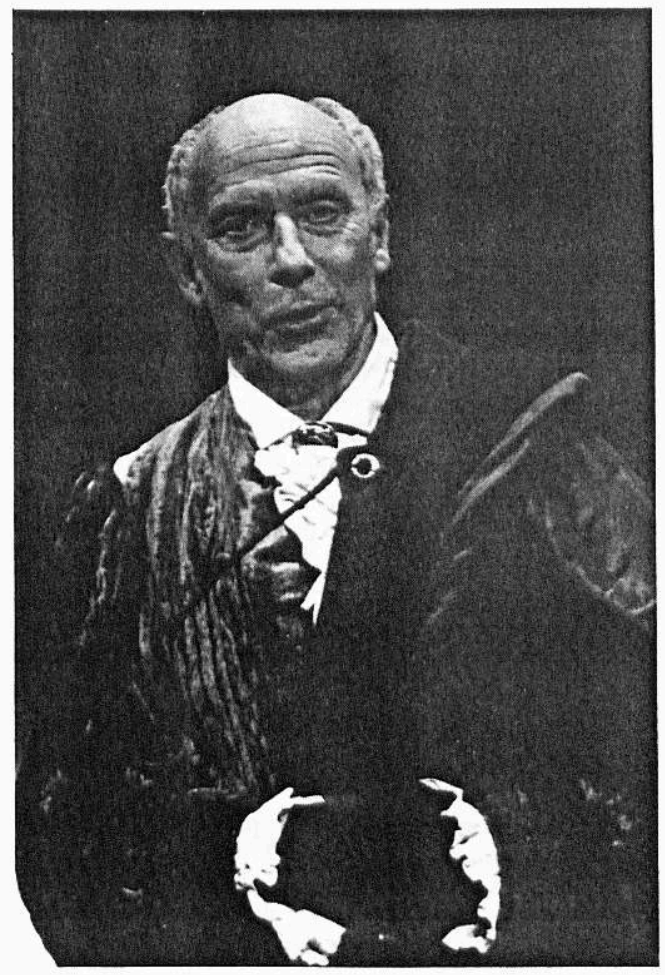

- Où jouiez-vous ?

- Un peu partout en Bourgogne, dans les villes et les villages et à Dijon.

- Comment étiez-vous accueillis par les gens du lieu?

- Très bien. A Pernand, nous avons trouvé plus d'une fois, quand nous revenions de jouer, des légumes et des œufs devant notre porte. Nous étions souvent invités à dîner, car tout le monde savait que nos ressources étaient modiques. 
- Vous avez dû laisser beaucoup d'amis en Bourgogne?

- Oui, et je les revois toujours avec plaisir.

- Votre compagnie a-t-elle évolué?

- Oui, lorsque, grâce à la loi Malraux de 1959, nous avons obtenu une subvention. Nous étions alors vingt-cinq comédiens. Nous invitions des metteurs en scène comme Jorge LAVELLI, Jean-Pierre VINCENT, Pierre VIAL, Roland MONOD, André STEIGER. Jorge LAVELLI a monté Yvonne, princesse de Bourgogne de Gombrowicz qui a été ensuite jouée chez Jean-Louis BARRAULT. Nous montions trois pièces par an que nous jouions de cinquante à quatre-vingt fois.

- Vos mises en scène ressemblaient-elles à celles de Jean VILAR ?

- Oui, au début. Nous étions fidèles à l'esthétique du plateau nu. Cette quasi-absence de décor facilitait nos déplacements car nous partions souvent en tournée et elle avait l'avantage d'être économique. Nous nous sommes ensuite davantage efforcés d'adapter le décor à chaque pièce.

- Après le théâtre de Bourgogne, vous êtes nommé en 1971 directeur du Théâtre National de Strasbourg.

- Oui, c'est Jacques DUHAMEL qui m'y a appelé.

- Quelle était la physionomie du T.N.S. quand vous y êtes arrivé ?

- Le T.N.S. était lourd car il employait cent personnes dans ses différents services. Il n'y avait que deux ou trois acteurs permanents. Les autres étaient engagés pour chaque spectacle. Il y avait aussi une école fondée par Michel SAINT-DENIS, neveu de COPEAU. Il y tenait beaucoup.

- Ce désir d'avoir une école a été celui de nombreux hommes de théâtre.

- Oui, il a été celui de CopeAu, de Dullin, de CHEREAu aux Amandiers et de Jean-Pierre VINCENT qui a enseigné au Conservatoire. Chacun d'eux a éprouvé le besoin d'avoir des disciples et le contact avec de jeunes comédiens est très important pour nous.

- Quel était le climat du T.N.S. ?

- Le climat n'était pas très bon, car 68 était encore tout proche. Le personnel était très divisé.

- Par quoi avez-vous commencé au T.N.S. ?

- J'ai voulu tout d'abord calmer les esprits. Le comédien s'engage, lorsqu'il joue, de tout son être. Il expose sa personne profondément. Il a besoin de se sentir entouré d'une grande fraternité. Autrement des blocages se créent. Il faut aussi qu'avant d'entrer dans un théâtre qui est une microsociété, le spectateur perçoive le climat qui $\mathrm{y}$ règne. Il doit entrer dans un théâtre comme dans un temple, avec un rituel.

- II me semble que vous rejoignez stanisLavski ${ }^{5}$ qui voulait absolument purifier le théâtre.

- Oui ; il faut toujours y revenir.

- Qu'avez-vous monté à Strasbourg ?

- Des pièces de LABICHE, de Pablo NERUDA, et Macbeth, mis en scène par Roger BLIN.

- Combien de temps êtes-vous resté à Strasbourg?

- Deux ans. En 1972 j'ai décidé de partir. 
- Pouvez-vous m'indiquer les raisons de cette décision?

- Je connaissais la réussite artistique et sociale. J'avais cependant l'impression de me fourvoyer. Lorsque j'étais jeune, j'espérais atteindre par la vie artistique la liberté et la compréhension du monde. Il m'a semblé que quelque chose de faux s'était glissé dans ma vie et que je passais à côté de ma finalité d'homme. La position sociale n'est pas la réalité d'un être humain.

- Où êtes-vous allé ?

- Comme Alceste, je me suis retiré dans le désert. Je suis allé dans le Sud tunisien et en Inde. Je revenais de temps en temps travailler en France.

- Quel était exactement votre but?

- Je voulais prendre du recul vis-à-vis de moi-même et savoir où était la vérité.

- Avez-vous rencontré des penseurs qui vous ont particulièrement marqué ?

- Oui, j'ai rencontré des disciples de Sri AUROBINDO, qui était décédé en 1950. Ils ont rendu vivant pour moi l'enseignement de leur maître.

- Puis-je vous demander ce que vous avez retiré de ces séjours en Inde?

- Ils m'ont aidé à me purifier. Ils m'ont révélé que la vérité n'était pas en dehors de moi.

- Je crois que c'est la leçon du brahmanisme. C'est en descendant en soi qu'on découvre le monde.

- Oui ; c'est cela.

- Et après l'Inde?

- Je suis venu à Besançon en 1979, au Centre de rencontres.

- Aviez-vous des raisons particulières ?

- J'avais travaillé une saison entière, en 1976, au Centre dramatique national de Besançon et il me semblait que je me plairais dans cette ville. Je serais à la disposition de ceux qui aiment le théâtre et veulent aller appréhender la nature profonde de l'être humain. J'ai rencontré à Besançon Jacques VINGLER qui a beaucoup œuvré pour l'éducation populaire et pour le théâtre amateur.

- Vous vous consacrez donc à l'enseignement théâtral sous toutes ses formes. Etes-vous toujours acteur et metteur en scène?

- Non, mais comme je travaille avec des groupes divers, je me considère toujours comme chef d'une troupe, même si celle-ci est un peu éclatée.

- Pouvez-vous me dire en quoi consiste votre enseignement?

- Il faut d'abord partir de l'idée que le théâtre sert à appréhender la connaissance. La relation entre le comédien et le spectateur doit être complète, pure, exigeante. L'homme qui est en face de moi sur la scène est le même que moi. Il me délivre un message que je vis.

- Quel est donc votre but, en tant que professeur d'art dramatique?

- Je m'efforce de mettre le comédien en état de création par un entraînement rigoureux et sévère. Nos possibilités sont immenses. Nous n'utilisons que $5 \%$ des possibilités de notre cortex supérieur qui est un superordinateur et qui dispose d'une capacité de progresser infinie. Celles-ci se réaliseront peut-être petit à petit dans un avenir lointain, dans mille ou deux mille ans. Si nous parvenons à mettre le cortex 
dans un état neutre, à le rendre disponible dans sa réception, il a la capacité de trouver une réponse à ce qui lui est proposé.

- Vous avez, me semble-t-il, des compétences scientifiques.

- Je me suis intéressé à des travaux de psychologues et à ceux du professeur FINDELKRAIS, physicien et biologiste, à partir de 1977.

- Y a-t-il là encore un rapport avec l'Orient ?

- Oui ; il faut arriver à un état de contemplation comme en Orient, à un vide qui n'est pas un néant, mais bien au contraire un mouvement.

- Lorsqu'un jeune comédien se trouve devant vous que lui conseillez-vous?

- Le comédien doit être en état de parfaite relaxation physique et mentale, il faut qu'il soit en relation avec ses sensations physiques du moment et en parfait éveil sur ce qui se passe en lui. Alors, devant le texte, la réponse doit venir. On ne joue pas ; on laisse jouer. On est dans la fiction avec le personnage et dans la réalité avec sa personne ; la conscience artistique fait le lien entre les deux termes.

- Vous faites certainement travailler à vos élèves la voix et le geste?

- Oui, bien sûr, mais il faut qu'ils aient, ne fût-ce que quelques secondes, l'expérience que je cherche à leur faire découvrir et qui est susceptible d'ouvrir la voie à d'autres progrès. Le jeune comédien se situe par rapport à cette présence qui le met en sécurité, la présence de l'état de création. Et c'est cette présence en lui qui crée sa présence pour le spectateur. Il nous appartient de détecter ce don.

\section{NOTES}

1. Sur Jean VILAR, cf. Alfred SIMON, Jean Vilar, La Manufacture, 1987.

2. Après la fermeture du Vieux Colombier en 1924, Jacques COPEAU forma avec un certain nombre de ses acteurs une troupe qui prit le nom de Compagnie des Copiaux. Ils jouèrent en Bourgogne de 1924 à 1929. Ils s'installèrent d'abord au château de Morteuil dans les environs de Beaune, en Saône et Loire, puis en 1925 à Pernand-Vergelesses en Côte d'Or où Jacques Copeau acheta une maison. Sur les Copiaux, cf. Le Journal de bord des Copiaus, présenté par Denis GONTARD, Seghers, 1974.

3. Les comédiens touchent actuellement une indemnité de chômage lorsqu'ils peuvent prouver qu'ils ont travaillé cinq-cent-huit heures dans une année. Un certain nombre d'entre eux jugent ce système insuffisant.

4. Jacques DUHAMEL était à cette époque Ministre à la Culture et Maire de Dole.

5. Notes Coulisses n ${ }^{\circ} 4$ p. 41 : Article sur K.C. STANISLAVSKI. 


\section{AUTEUR}

\section{PHILIPPE BARON}

Professeur de Littérature française à l'Université de Franche-Comté, il est l'auteur d'une thèse

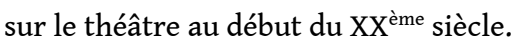

